\title{
MDA-7/IL-24 suppresses tumor adhesion and invasive potential in hepatocellular carcinoma cell lines
}

\author{
WEI HUO ${ }^{1,2}$, ZHI-MIN LI ${ }^{1}$, XIAO-MIN ZHU ${ }^{1}$, YONG-MING BAO ${ }^{2}$ and LI-JIA AN ${ }^{2}$ \\ ${ }^{1}$ Department of Medical Oncology, Dalian Municipal Central Hospital, Dalian, Liaoning 116033; \\ ${ }^{2}$ School of Life Science and Biotechnology, Dalian University of Technology, Dalian, Liaoning 116024, P.R. China
}

Received January 13, 2013; Accepted April 12, 2013

DOI: $10.3892 /$ or.2013.2507

\begin{abstract}
Melanoma differentiation associated gene-7 (MDA-7)/interleukin-24(IL-24) has been considered as a tumorsuppressor gene, which suppresses the growth and induces the apoptosis of cancer cells. In the present study, we investigated the effect and mechanisms of MDA-7/IL-24 regarding the inhibition of metastasis of HepG2 and BEL-7402 human hepatocellular carcinoma (HCC) cells in vitro. We established MDA-7/IL-24-overexpressing HepG2 and BEL-7402 cell lines and found that MDA-7/IL-24 overexpression inhibited tumor cell adhesion and invasion, and induced $\mathrm{G} 2 / \mathrm{M}$ arrest in tumor cells. To explore its mechanism of action, western blotting and real-time-PCR assay were used to investigate the expression of E-cadherin, CD44, ICAM-1, matrix metalloproteinase (MMP)-2 and -9, CyclinB, Twist, survivin, p-ERK and p-Akt. ELISA assay was used to measure the secretion of TGF- $\beta$, and a reporter gene assay was used to detected the transcriptional activity of NF- $\kappa \mathrm{B}$ and AP-1 in HepG2 and BEL-7402 cells. The results showed that MDA-7/IL-24 overexpression decreased the expression of CD44, ICAM-1, MMP-2/-9, CyclinB, Twist, survivin, TGF- $\beta$ and $\mathrm{p}-\mathrm{Akt}$, transcriptional activity of $\mathrm{NF}-\kappa \mathrm{B}$, and increased the expression of E-cadherin and $\mathrm{p}-\mathrm{ERK}$ and transcriptional activity of AP-1 in HepG2 and BEL-7402 cells. Our results revealed that MDA-7/IL-24 mediated the inhibition of adhesion and invasion in HepG 2 and BEL-7402 cells by suppressing metastasis-related gene expression. Thus, MDA-7/ IL-24 may be used as a novel cancer-suppressor gene for the therapy of human HCC.
\end{abstract}

\section{Introduction}

Hepatocellular carcinoma (HCC) is one of the most common causes of cancer-related mortality and exhibits high metastatic

Correspondence to: Dr Li-Jia An, School of Life Science and Biotechnology, Dalian University of Technology, Dalian, Liaoning 116024, P.R. China

E-mail: ljan.bio54@yahoo.cn

Key words: human hepatocellular carcinoma, MDA-7/IL-24, tumor metastasis potential and is associated with poor patient prognosis. More than 700,000 cases of HCC were diagnosed in 2008 (1-3). Melanoma differentiation associated gene-7 (MDA-7)/ interleukin-24 (IL-24) is a member of the IL-10 gene family, and several reports have indicated that MDA-7/IL-24 overexpression causes tumor growth suppression and tumor cell apoptosis in mesotheliomas, osteosarcoma, melanoma, lung cancer, breast cancer, pancreatic cancer, glioblastoma and prostate cancer (4-16), suggesting that MDA-7/IL-24 may prove to be a potential strategy for cancer therapy. However, the mechanisms through which MDA-7 expression exerts its anti-neoplastic activity, tumor-specificity and efficacy across a spectrum of human cancer types have yet to be fully elucidated. We, therefore, aimed to investigate the effect of the ectopic production of MDA-7/IL-24 on the metastasis of HCC HepG2 and BEL-7402 cells in vitro and attempted to identify the underlying mechanisms involved in its suppression of metastasis.

In the present study, we demonstrated for the first time that MDA-7/IL-24 inhibits the adhesion and invasion of HCC HepG2 and BEL-7402 cells by downregulating the expression of CD44, ICAM-1, CyclinB, Twist, survivin, p-Akt and matrix metalloproteinases (MMPs), and by upregulating the expression of E-cadherin and p-ERK. Furthermore, we confirmed that MDA-7/IL-24 reduced the secretion of TGF- $\beta$ and the transcriptional activity of $\mathrm{NF}-\kappa \mathrm{B}$ and increased the transcriptional activity of AP-1 in HepG2 and BEL-7402 cells. Thus, MDA-7/IL-24 may provide multiple benefits as an anticancer therapeutic strategy due to its inhibition of tumor metastasis.

\section{Materials and methods}

Cell culture. Human HCC cell lines HepG2 and BEL-7402 were obtained from the American Type Culture Collection (ATCC; Manassas, VA, USA), and the cell lines were cultured in Dulbecco's modified Eagle's medium (DMEM) (10\% FBS) under conditions of $37^{\circ} \mathrm{C}$ and $5 \% \mathrm{CO}_{2}$.

Plasmid construction. The mda-7/IL-24 coding sequences were amplified by RT-PCR. The primers were: sense 1, 5'-AATAGGGCTAGCGCCACCATGAATTTTCAACAGAG GCT-3' and sense 2, 5'-GAATTCGGTCTCCTCGAGGAGC TTGTAGAATTTCTGCA-3' and were verified and ligated into the pcDNA3.1 vector (Invitrogen). 
Transfection of Mda-7/IL-24. The pcDNA3.1 empty vector plasmid and pcDNA3.1-mda-7/IL-24 expression plasmid were transfected into HepG2 and BEL-7402 cells. A pool of transfectants was selected using G418 to build the mda-7/IL-24overexpressing sublines (Ad.mda-7-1 and Ad.mda-7-2) and the negative control empty vector subline (Ad.vec).

Adhesion assay. Cells were cultured in Matrigel-coated 24-well plates (Collaborative Biomedical), incubated, and washed with cold PBS $6 \mathrm{~h}$ later. The adhesion assay was performed by MTS assay at $490 \mathrm{~nm}$. The cell adhesion rate was calculated by the absorption of the MDA-7/IL-24-overexpressing group or negative control group/the absorption of the parent group.

Cell invasion assay. Cells were harvested, resuspended in serum-free DMEM, and then transferred to hydrated Matrigel chambers $(25,000$ cells/well) of the Transwell system. The chambers were then incubated in DMEM with $10 \%$ FBS in the bottom chambers before examination overnight. The cells on the upper surface were scraped and washed away, whereas the invaded cells in the lower surface were fixed, stained and counted under a microscope, and the relative number was calculated (magnification, x20).

Cell cycle analysis. Cells were cultured, treated with serumfree DMEM for $24 \mathrm{~h}$ for synchronization, and ixed in $70 \%$ ethanol at $4^{\circ} \mathrm{C}$ overnight. Cells were then resuspended and incubated with $1 \mathrm{mg} / \mathrm{ml}$ of RNase A and $0.5 \mathrm{mg} / \mathrm{ml}$ propidium iodide for $30 \mathrm{~min}$ in the dark. Cell cycle analysis was analyzed by flow cytometry, and the percentage of cells at each phase of the cell cycle was determined using MultiCycle software.

ELISA assay. The cell culture supernatant was collected and added to a microplate. The anti-TGF- $\beta$ antibody was then added and incubation was carried out at $37^{\circ} \mathrm{C}$ for $30 \mathrm{~min}$. HRP was added and incubation was carried out at $37^{\circ} \mathrm{C}$ for 30 min after washing with wash buffer. TMB was added and incubated at room temperature for $20 \mathrm{~min}$ in the dark. The absorbance was read at $450 \mathrm{~nm}$ after the stop solution was added, and the TGF- $\beta$ content was calculated using a standard curve.

Real-time quantitative PCR. Total RNA was isolated by TRIzol assay, and real-time-PCR assays were performed by SYBR-Green incorporation. The relative gene expression was determined by duct calculation utilizing actin for normalization. GAPDH was used as a reference gene. The following primers were used to amplify cDNA fragments: human E-cadherin, (forward) 5'-CAGCATCACTGGCCAAGGAGC TGA-3' and (reverse) 5'-GACCACACTGATGACTCCTGT GTTCC-3'; humanCD44,(forward) 5'-CGTGATGGCACCCGC TATGT-3' and (reverse) 5'-CAGGGATTCTGTCTGTGCTG TCG-3'; human ICAM-1, (forward) 5'-CTCCAATGTGCCA GGCTTG-3' and (reverse) 5'-CAGTGGGAAAGTGCCAT CCT-3'; human MMP-2, (forward) 5'-CTTCCAAGTCTGGAG CGATGT-3' and (reverse) 5'-TACCGTCAAAGGGGTATC CAT-3'; human MMP-9, (forward) 5'-GGGACGCAGACATC GTCATC-3' and (reverse) 5'-TCGTCATCGTCGAAATG GGC-3'; human CyclinB1, (forward) 5'-GGCCAAAATGC CTATGAAGA-3' and (reverse) 5'-AGATGTTTCCATTGGGC

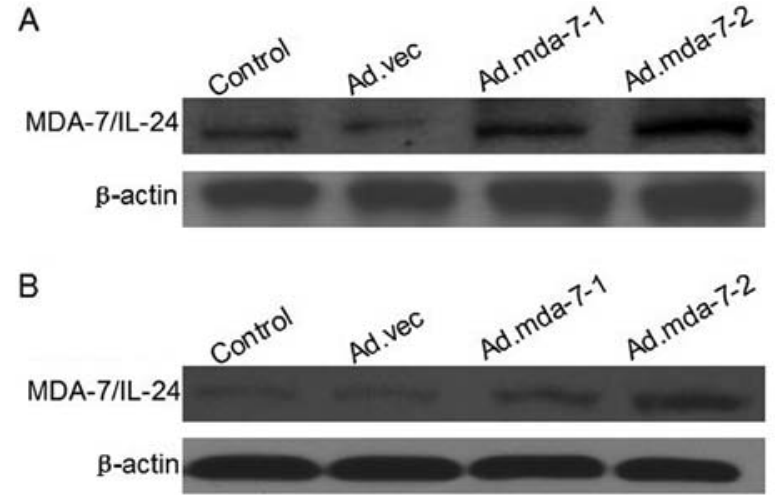

Figure 1. Expression of MDA-7/IL-24 in the establishment of stable HepG2 and BEL-7402 cells. Western blot analysis was used to measure the level of MDA-7/IL-24 expression in (A) HepG2 and (B) BEL-7402 cells MDA-7/IL-24 was overexpressed in the cell lines transfected with the Ad.mda-7 expression plasmid.

TTG-3'; human Twist, (forward) 5'-AGTCCGCAGTCTTAC GAGGA-3' and (reverse) 5'-GCAGAGGTGTGAGGATG GT-3'; human survivin, (forward) 5'-GCCCAGTGTTTCTT CTGCTT-3' and (reverse) 5'-CCGGACGAATGCTTTTTA TG-3'; human GAPDH (forward) 5'-TGTTGCCATCAATGAC CCCTT-3' and (reverse) 5'-CTCCACGACGTACTCAGCG-3.

Western blot analysis. The cells were lysed and analyzed using 12\% SDS-PAGE. The proteins were transferred to polyvinylidene difluoride membranes and blocked at $4^{\circ} \mathrm{C}$ overnight with $5 \%$ milk and incubated with the monoclonal antibodies against: MDA-7/IL-24 (1:800), ICAM-1 (1:800), MMP-2 (1:800), MMP-9 (1:500), CyclinB (1:500), E-cadherin (1:800), CD44 (1:500), Twist (1:500), survivin (1:500), p-ERK (1:800), ERK (1:800), p-Akt (1:800), Akt (1:800) and mouse monoclonal $\beta$-actin $(1: 2,000)$. The results were visualization using a chemiluminescence detection kit.

Reporter gene assay. Cells were transfected with pAP-1-luc or pNFkB-luc and pRL-SV40 and plated in a 24-well plate at $1 \times 10^{5}$ cells/well at $37^{\circ} \mathrm{C}$. The cells were then counted by luciferase assay after $24 \mathrm{~h}$.

Statistical analysis. Data are presented as the means \pm standard deviation (SD) and were evaluated with SPSS 11.0. Analysis of variance was performed by one-way ANOVA, and differences were considered significant at $\mathrm{P}<0.05$.

\section{Results}

Expression of the MDA-7/IL-24 gene in the establishment of stable HepG2 and BEL-7402 cells. The transfection efficiency of HepG2 and BEL-7402 cells was measured by western blotting. The protein expression in the Ad.mda-7-1 and Ad.mda-7-2 groups was higher when compared with the expression in the Ad.vec and parent groups (Fig. 1).

MDA-7/IL-24 inhibits tumor cell adhesion and invasive potential in HepG2 and BEL-7402 cells. We assessed the ability of MDA-7/IL-24 to inhibit cell adhesion in HepG2 and 
A
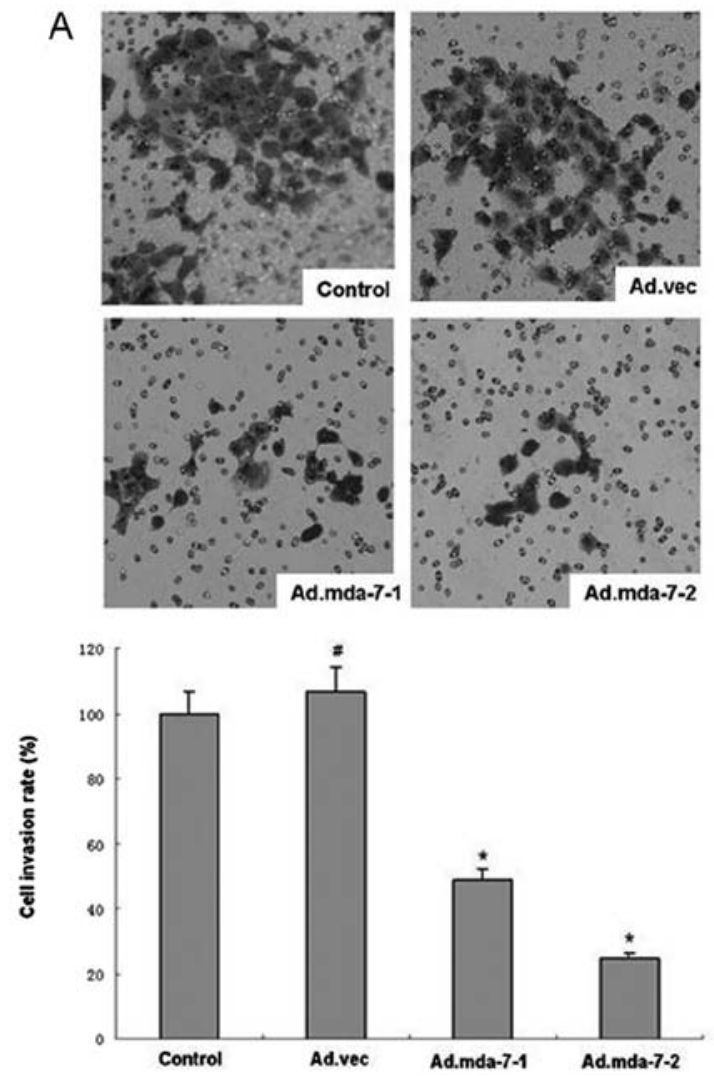

B
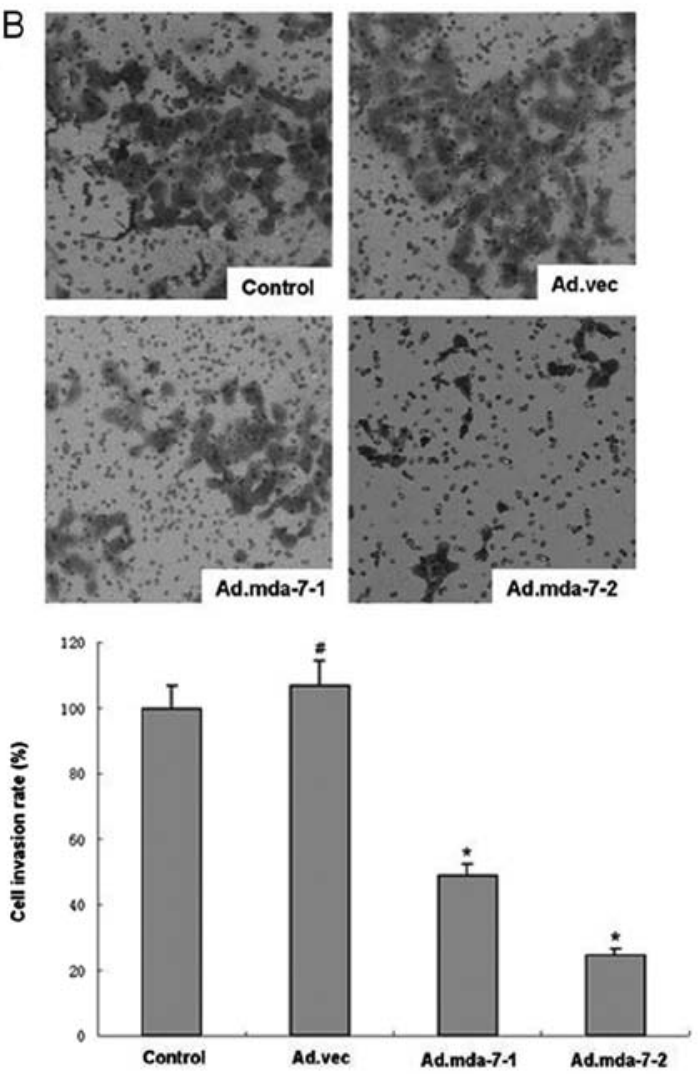

Figure 2. MDA-7/IL-24 inhibits tumor cell invasive potential in HepG2 and BEL-7402 cells. Quantitative analysis revealed a decreased rate of cell invasion in the (A) HepG2 and (B) BEL-7402 cells in the MDA-7/IL-24-overexpressing groups. Values are shown as the means \pm SD $\left({ }^{\#} \mathrm{P}>0.05\right.$, $\left.{ }^{*} \mathrm{P}<0.05\right)$.
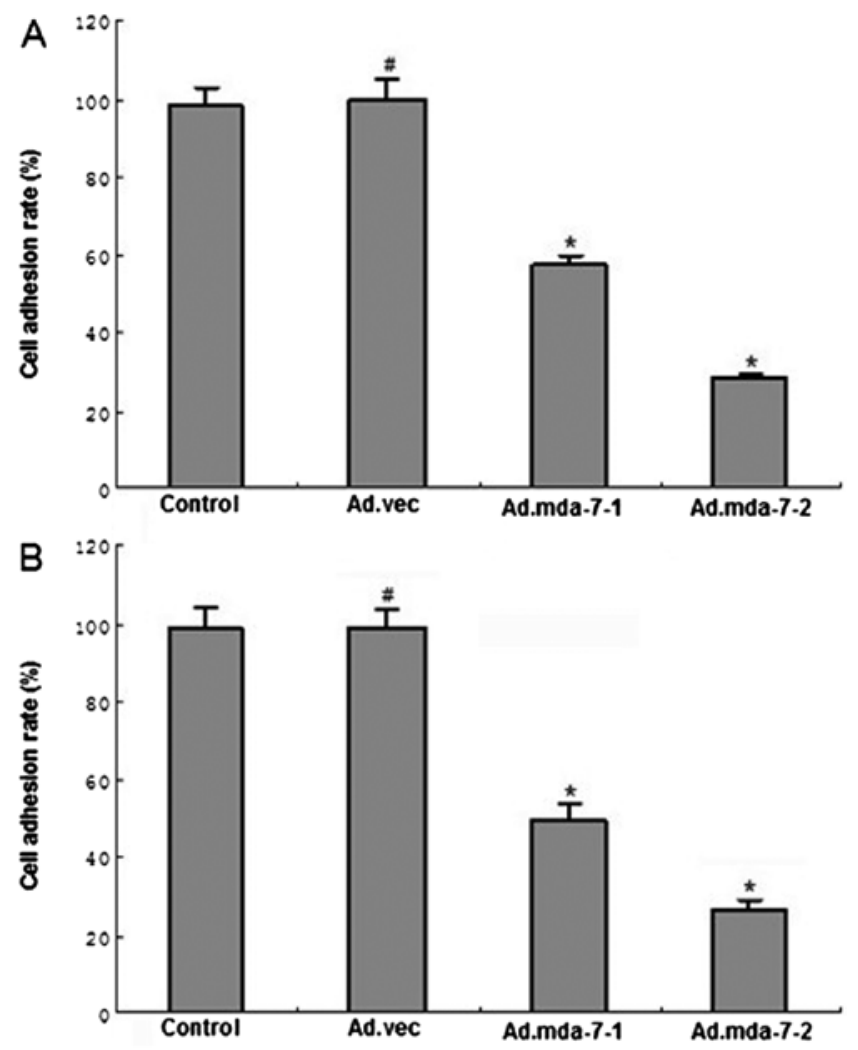

Figure 3. MDA-7/IL-24 inhibits tumor cell adhesion in the HepG2 and BEL7402 cells. Quantitative analysis showed a decreased rate of cell adhesion in the (A) HepG2 and (B) BEL-7402 cells in the MDA-7/IL-24-overexpressing groups. Values are shown as means $\pm \mathrm{SD}\left({ }^{\sharp} \mathrm{P}>0.05,{ }^{*} \mathrm{P}<0.05\right)$.
BEL-7402 cell lines. HCC cells overexpressing MDA-7/IL-24 were significantly less able to adhere when compared to the empty vector or control groups (Fig. 3). Furthermore, HepG2 and BEL-7402 cells overexpressing MDA-7/IL-24 were much less invasive than that of the empty vector or control groups (Fig. 2), indicating that MDA-7/IL-24 effectively inhibits tumor cell adhesion and invasive potential in HepG2 and BEL-7402 cells.

MDA-7/IL-24 regulates expression of metastasis-related genes in HepG2 and BEL-7402 cells. We next examined the regulatory effect of MDA-7/IL-24 on tumor metastasis-related genes which influence tumor adhesion and invasion by western blot analysis and RT-PCR assay. The results showed that the expression levels of CD44, ICAM-1, MMP-2/-9, CyclinB, Twist, survivin and p-Akt in MDA-7/IL-24-overexpressing cells were significantly decreased when compare to these levels in the empty vector or control groups at the transcription and translation levels. Moreover, the expression of E-cadherin and p-ERK was significantly increased (Figs. 4 and 5). The results revealed that MDA-7/IL-24 inhibits expression of tumor metastasis-related genes in HepG2 and BEL-7402 cells.

MDA-7/IL-24 inhibits the TGF- $\beta$ production in tumor cells. We used ELISA assay to detect the expression of TGF- $\beta$. Results showed that production of TGF- $\beta$ was decreased in the HepG2 and BEL-7402 cells overexpressing MDA-7/IL-24, when compared with the production in the empty vector negative control or parent groups (Fig. 6). 
A

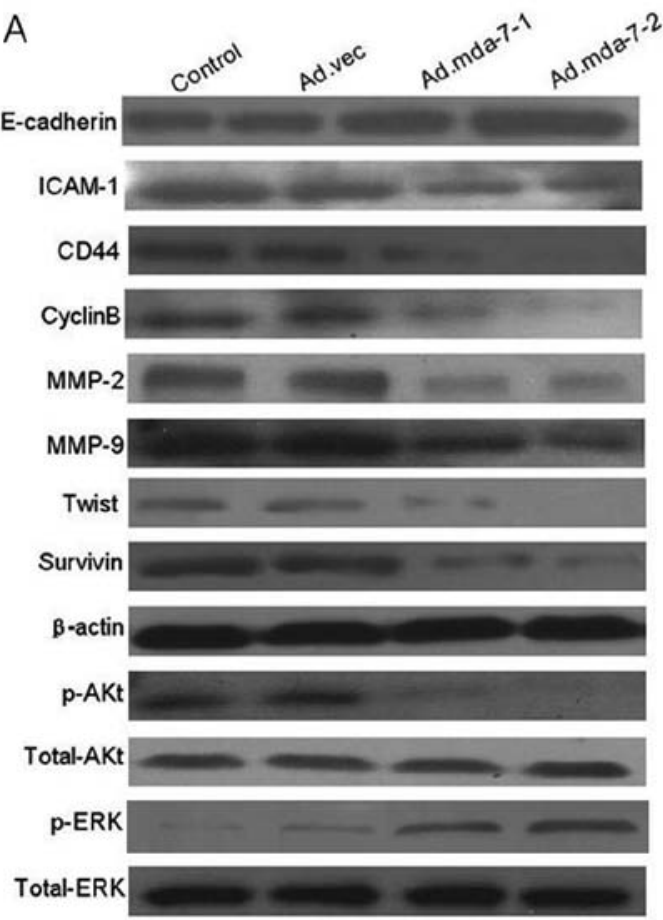

B

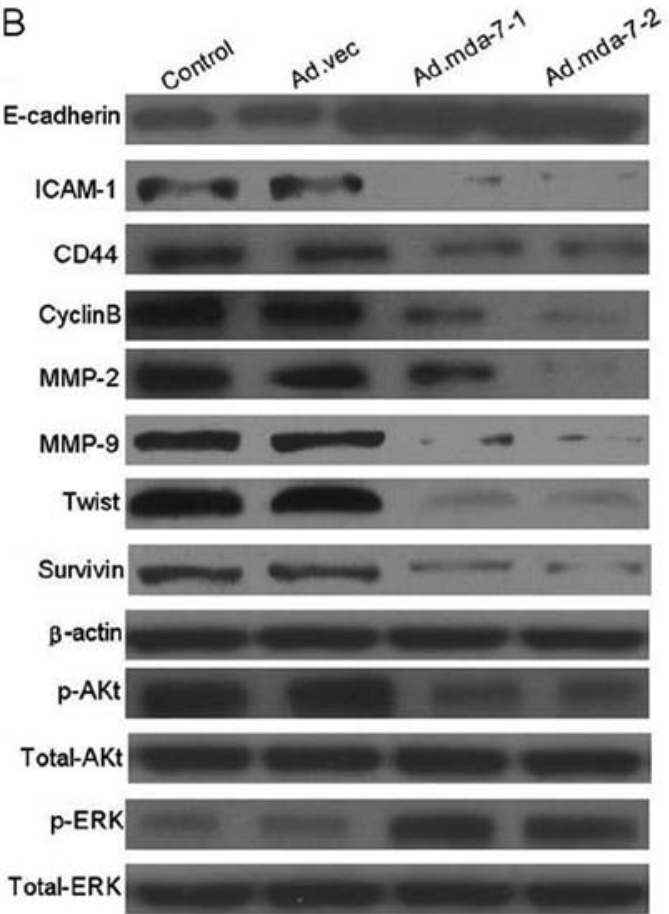

Figure 4. MDA-7/IL-24 regulates metastasis-related gene protein expression in HepG2 and BEL-7402 cells. Western blot assay was used to assess the expression of metastasis-related genes in HepG2 and BEL-7402 cells. The protein expression of CD44, ICAM-1, MMP-2/-9, CyclinB, Twist, survivin and p-Akt in the (A) HepG2 and (B) BEL-7402 cells overexpressing MDA-7/IL-24 was significantly decreased and the expression of E-cadherin and p-ERK was significantly increased when compared to the levels in the empty vector or control groups. $\beta$-actin was used as the internal loading control.
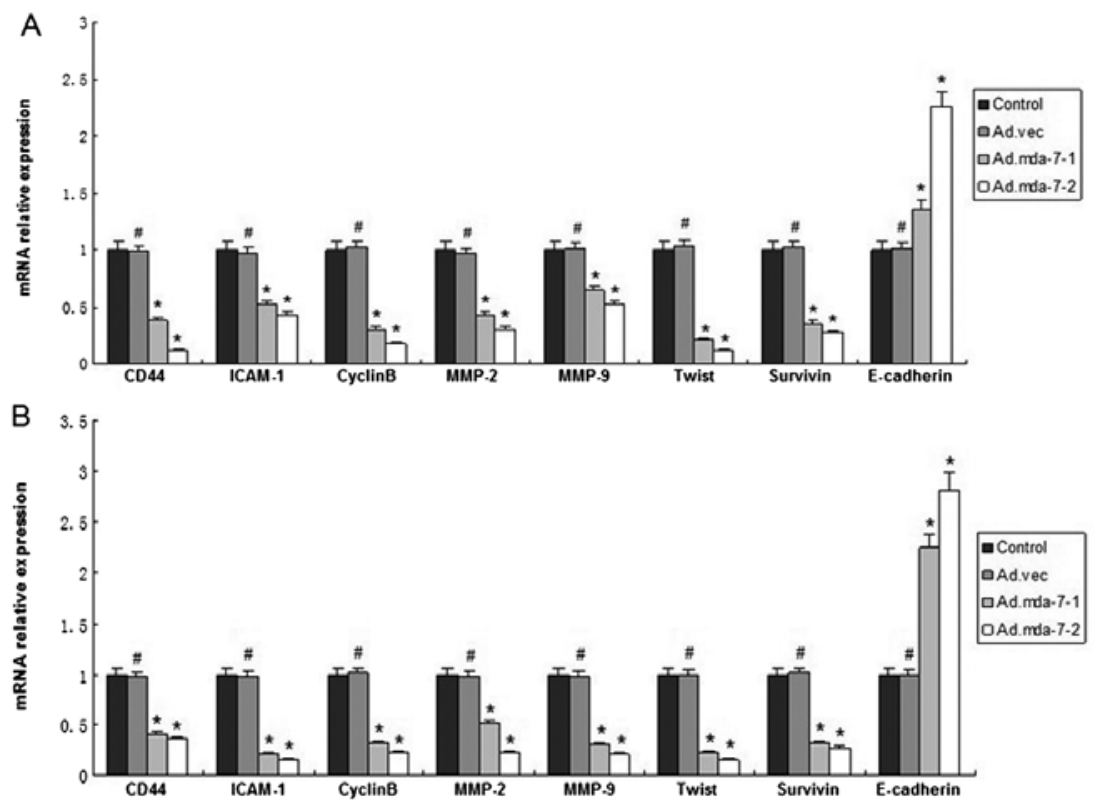

Figure 5. MDA-7/IL-24 regulates metastasis-related gene mRNA expression in HepG2 and BEL-7402 cells. The mRNA expression of metastasis-related genes was analyzed by RT-PCR. The mRNA expression of CD44, ICAM-1, MMP-2/-9, CyclinB, Twist and survivin in the (A) HepG2 and (B) BEL-7402 cells overexpressing MDA-7/IL-24 was significantly decreased and expression of E-cadherin was increased significantly when compared to the empty vector or control groups. GAPDH was used as the internal loading control. Values are shown as means $\pm \mathrm{SD}\left({ }^{*} \mathrm{P}>0.05,{ }^{*} \mathrm{P}<0.05\right)$.

MDA-7/IL-24 induces accumulation of tumor cells in the G2/M phase of the cell cycle. As shown in Fig. 7, MDA-7/IL-24 induced the accumulation of HepG2 and BEL-7402 cells in the $\mathrm{G} 2 / \mathrm{M}$ phase. We found that the percentages of $\mathrm{HepG} 2$ and BEL-7402 cells in the G2/M phase were 11.26 and $14.62 \%$ in the MDA-7/IL-24-overexpressing groups, $7.25 \%$ in the empty vector group and $6.74 \%$ in the control group $(\mathrm{P}<0.01)$, while the percentages of HepG2 and BEL-7402 cells in the S phase were 38.75 and $41.25 \%$ in the MDA-7/IL-24-overexpressing groups, $30.06 \%$ in the empty vector group and $28.13 \%$ in the 

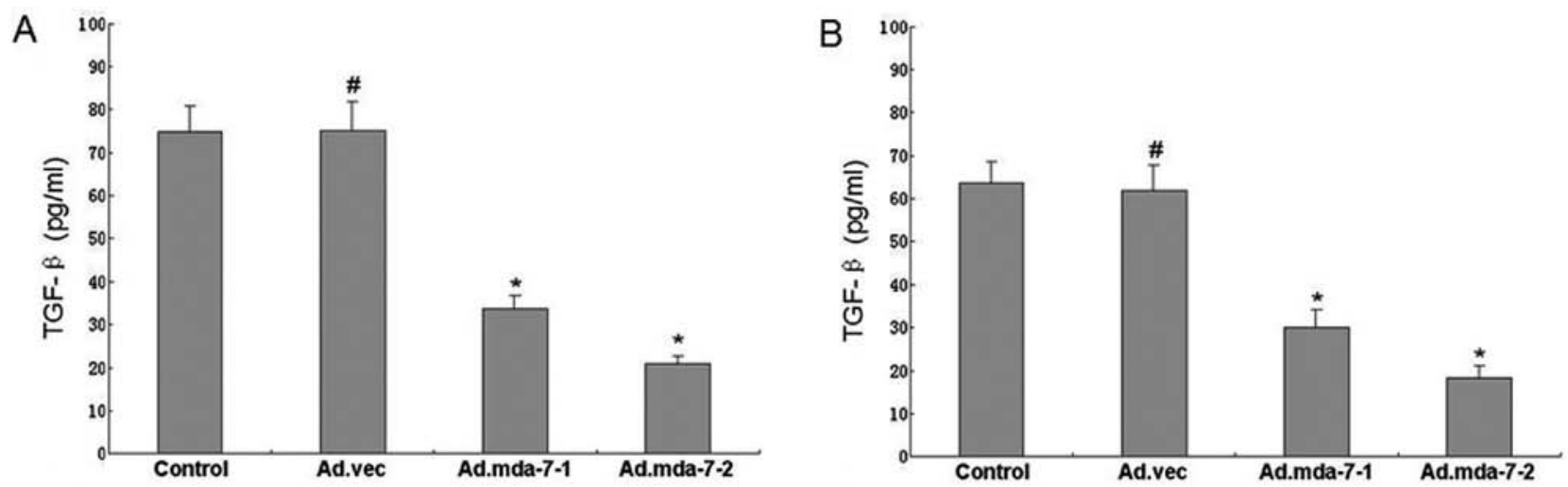

Figure 6. Inhibition of TGF- $\beta$ production in HepG2 and BEL-7402 cells by MDA-7/IL-24. ELISA assay was used to detect the TGF- $\beta$ production. The production of TGF- $\beta$ was decreased in (A) HepG2 and (B) BEL-7402 cells overexpressing MDA-7/IL-24 when compared with the empty vector or control groups. Values are shown as means $\pm \mathrm{SD}\left({ }^{*} \mathrm{P}>0.05,{ }^{*} \mathrm{P}<0.05\right)$.

A
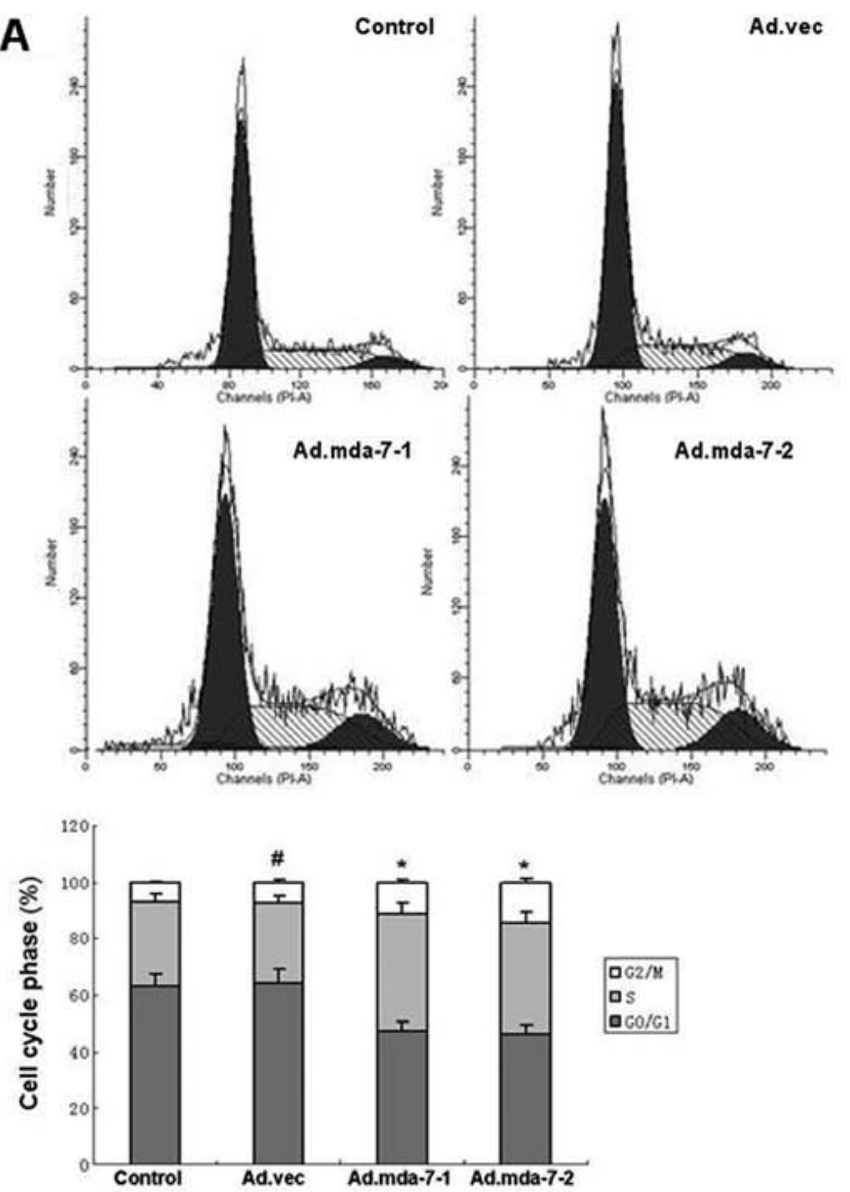

B
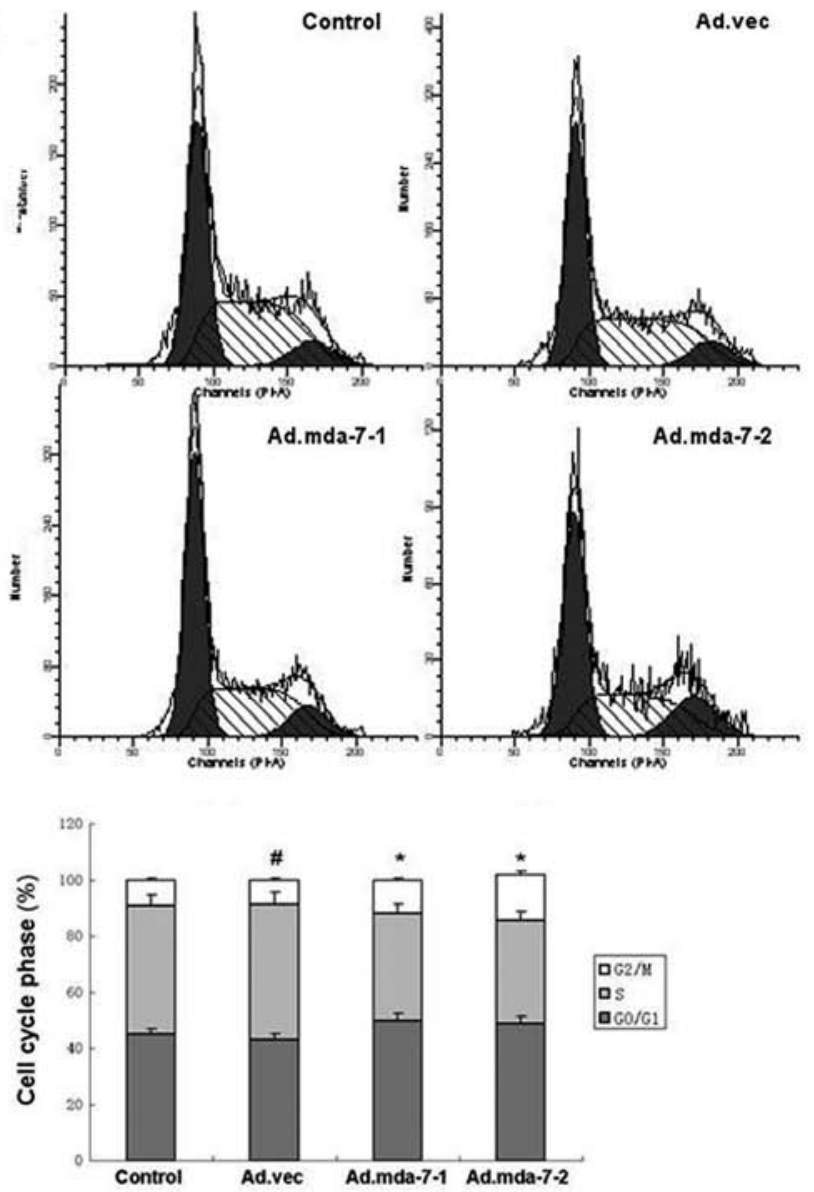

Figure 7. Effect of MDA-7/IL-24 on cell cycle distribution in HepG2 and BEL-7402 cells. The effect of MDA-7/IL-24 on cell cycle distribution in HepG2 and BEL-7402 cells was measured by flow cytometry. The proportions of (A) HepG2 and (B) BEL-7402 cells overexpressing MDA-7/IL-24 in the G2/M phase were significantly higher than the proportions in the empty vector or control groups. Values are shown as mean $\pm \mathrm{SD}\left({ }^{\#} \mathrm{P}>0.05,{ }^{*} \mathrm{P}<0.05\right)$.

control group $(\mathrm{P}<0.01)$. The results revealed that MDA-7/IL-24 induced an accumulation of HepG2 and BEL-7402 cells in the G2/M phase.

MDA-7/IL-24 downregulates the transcriptional activation of AP- 1 and NF- $\kappa B$ in the HepG2 and BEL-7402 cells. We examined the effect of MDA-7/IL-24 on the transcriptional activation of AP-1 and NF-kB in HepG2 and BEL-7402 cells by luciferase reporter assay. We found that MDA-7/IL-24 downregulated the transcriptional activation of NF- $\mathrm{\kappa B}$ and upregulated the transcriptional activation of AP-1 in HepG2 and BEL-7402 cells (Fig. 8).

\section{Discussion}

MDA-7/IL-24 is a cytokine-like protein of the IL-10 cytokine family and has been reported to decrease survival in adjacent tumor cells while sparing normal cells (17-20). The present 

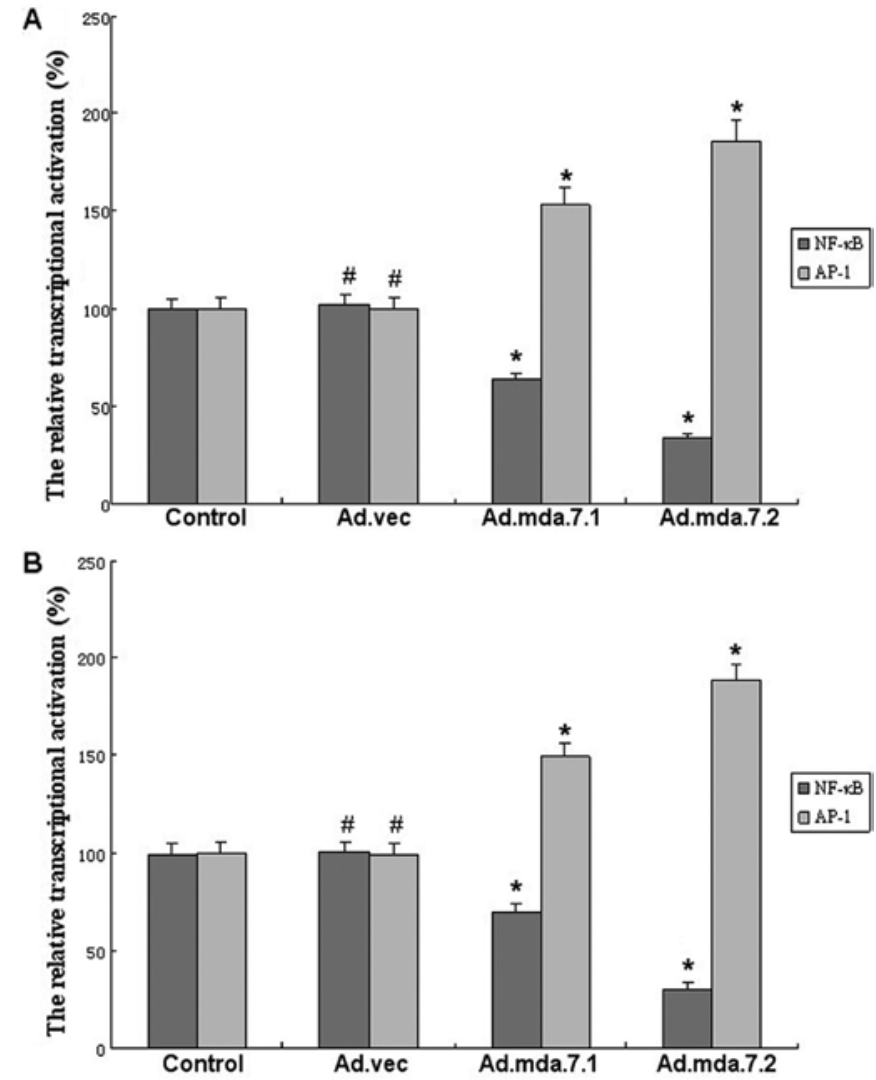

Figure 8. Effect of MDA-7/IL-24 on AP-1 and NF- $\mathrm{kB}$ transcriptional activation in HepG2 and BEL-7402 cells. The transcriptional activation of AP-1 and NF- $\mathrm{KB}$ was regulated in the (A) HepG2 and (B) BEL-7402 cells overexpressing MDA-7/IL-24, when compared with the empty vector or control groups by report gene assay. Values are shown as means $\pm \mathrm{SD}\left({ }^{\#} \mathrm{P}>0.05\right.$, $\left.{ }^{*} \mathrm{P}<0.05\right)$.

study adds to the literature findings regarding the metastatic inhibitory function of MDA-7/IL-24 in solid human malignancies, with particular reference to HCC. In the present study, the results revealed that MDA-7/IL-24 inhibited the potential of adhesion and invasion of human HCC HepG2 and BEL-7402 cells, and the invasion and adhesion inhibition ability of the cells was enhanced along with the increased expression of MDA-7/IL-24. Several reports have demonstrated that MDA-7/IL-24 overexpression results in growth inhibition and apoptosis in many types of cancer. MDA-7/IL-24 is considered as an ideal gene for tumor gene therapy due to its low deleterious effects on normal cells (21). We transfected the MDA-7/IL-24 expression plasmid into HepG2 and BEL-7402 cell lines, and western blot assay showed that MDA-7/IL-24 was overexpressed in cells transfected with Ad.mda-7-1 and Ad.mda-7-2, while the parental and Ad.vec groups did not show protein overexpression.

MDA-7/IL-24 induces tumor-suppressive activity through multiple mechanisms. In order to explore its mechanisms, we investigated the protein and mRNA expression of E-cadherin, CD44, ICAM-1, CyclinB, Twist, survivin, p-ERK and p-Akt in tumor cells. The results indicated that MDA-7/IL-24 overexpression decreased the expression of CD44, ICAM-1, CyclinB, Twist, survivin and p-Akt, while the expression of E-cadherin and p-ERK was upregulated.
E-cadherin, CD44 and ICAM-1 are known as significant adhesion molecules, and are necessary for cell adhesion, migration, proliferation, apoptosis and cell signal transmission (22). Overexpression of MDA-7/IL-24 induced the downregulation of CD44/ICAM-1 and the upregulation of E-cadherin, indicating that one mechanism involved in the suppression of tumor cell adhesion and invasion by MDA-7/IL-24 is through the regulation of the expression of adhesion molecules.

CyclinB is a key protein in cell cycle regulation by regulating cytoskeletal dynamics. Recent research suggests that CyclinB may regulate tumor growth, invasion and metastasis, and upregulation of CyclinB expression increases tumor metastasis potential. MDA-7/IL-24 induced the accumulation of HepG2 and BEL-7402 cells in the G2/M phase of the cell cycle. Twist is a transcription factor which relies on the basic DNA-binding region and the helix-loop-helix structure that allows monomers to form functional dimers that can identify and bind to the E-box DNA motif. Twist has been considered to inhibit E-cadherin expression, promote tumor cell adhesion and invasion. Survivin promotes cell adhesion and invasion by cell cycle-related kinases and regulation of the cell division process. Our results suggest that MDA-7/IL-24 suppresses tumor adhesion and invasion by controlling the expression of E-cadherin, CD44, ICAM-1, CyclinB, Twist and survivin and that together they are important to human HCC metastasis.

The activity of proteolysis enzymes is attributed to degradation of the tumor cell extracellular matrix (ECM). Among the proteases implicated in tumor cell dissemination are the MMPs (23), which are required by cells for tissue remodeling. However, the production of MMPs has been observed in many invasive tumor cell lines and during tumor growth (24). In fact, MMP-2/-9 levels appear to be prognostically significant during tumor progression in many tumor types $(11,25-27)$, and the extent of MMP overproduction correlates with prognosis. Therefore, we investigated and found that MDA-7/IL-24 overexpression decreased the expression of MMP-2/-9 at the protein and mRNA levels. Additionally, MDA-7/IL-24 influenced TGF- $\beta$ activity in tumor cells. ELISA assay was used to investigate the secretion of TGF- $\beta$ in HCC cancer cells, and we found that MDA-7/IL-24 induced the downregulation of TGF- $\beta$.

$\mathrm{NF}-\kappa \mathrm{B}$ is a transcription factor which upregulates many types of metastasis-related genes, and research has demonstrated that $\mathrm{NF}-\kappa \mathrm{B}$ regulates tumor metastasis potential. AP-1 is composed of the c-Jun and c-fos and also controls numerous genes contributing to the process of tumor metastasis. We hypothesized the regulatory role of MDA-7/IL-24 in the control of the NF- $\kappa \mathrm{B}$ and AP-1 transcription activation and demonstrated that MDA-7/IL-24 suppresses tumor metastasis potential by regulating their transcription activation.

Additionally, we explored the contribution of MDA-7/IL-24 on tumor metastasis signaling molecules. Numerous reports have shown that Akt and ERK are involved in the processes of adhesion and invasion in many types of tumors, and also control expression of transcription factors. In the present study, we examined the effect of MDA-7/IL-24 on the Akt and ERK pathway and demonstrated that MDA-7/IL-24 regulates Akt and ERK phosphorylation $(28,29)$. Therefore, we presumed that the modulation of the Akt and ERK signaling pathway contributing to the AP-1 and NF- $\kappa \mathrm{B}$ transcriptional activation 
regulation is the basis for the suppression of tumor metastasis by MDA-7/IL-24.

In conclusion, we demonstrated for the first time that MDA-7/IL-24 inhibits the metastatic potential of human HepG2 and BEL-7402 cells in vitro. Thus, MDA-7/IL-24 may provide an effective therapeutic strategy for HCC and may reduce tumor metastasis.

\section{References}

1. Ferlay J, Shin HR, Bray F, et al: Estimates of worldwide burden of cancer in 2008: GLOBOCAN 2008. Int J Cancer 127: 2893-2917, 2010.

2. Forner A, Llovet JM and Bruix J: Hepatocellular carcinoma. Lancet 379: 1245-1255, 2012.

3. Tung-Ping Poon R, Fan ST and Wong J: Risk factors, prevention, and management of postoperative recurrence after resection of hepatocellular carcinoma. Ann Surg 232: 10-24, 2000.

4. Fickenscher H, Hör S, Küpers H, et al: The interleukin-10 family of cytokines. Trends Immunol 23: 89-96, 2002.

5. Jiang H, Su ZZ, Lin JJ, et al: The melanoma differentiation associated gene mda-7 suppresses cancer cell growth. Proc Natl Acad Sci USA 93: 9160-9165, 1996.

6. Jiang H, Lin JJ, Su ZZ, et al: Subtraction hybridization identifies a novel melanoma differentiation associated gene, mda-7, modulated during human melanoma differentiation, growth and progression. Oncogene 11: 2477-2486, 1995.

7. Gopalkrishnan RV, Sauane M and Fisher PB: Cytokine and tumor cell apoptosis inducing activity of mda-7/IL-24. Int Immunopharmacol 4: 635-647, 2004.

8. Lebedeva IV, Sauane M, Gopalkrishnan RV, et al: mda-7/IL-24: exploiting cancer's Achilles' heel. Mol Ther 11: 4-18, 2005.

9. Allen M, Pratscher B, Roka F, et al: Loss of novel mda-7 splice variant (mda-7s) expression is associated with metastatic melanoma. J Invest Dermatol 123: 583-588, 2004.

10. Sarkar D, Su ZZ, Lebedeva IV, et al: mda-7 (IL-24) mediates selective apoptosis in human melanoma cells by inducing the coordinated overexpression of the GADD family of genes by means of p38 MAPK. Proc Natl Acad Sci USA 99: 10054-10059, 2002.

11. Ramesh R, Ito I, Gopalan B, Saito Y, et al: Ectopic production of MDA-7/IL-24 inhibits invasion and migration of human lung cancer cells. Mol Ther 9: 510-518, 2004.

12. McKenzie T, Liu Y, Fanale M, et al: Combination therapy of Ad-mda7 and trastuzumab increases cell death in Her-2/neu-overexpressing breast cancer cells. Surgery 136: 437-442, 2004.

13. Lebedeva IV, Su ZZ, Sarkar D, et al: Induction of reactive oxygen species renders mutant and wild-type K-ras pancreatic carcinoma cells susceptible to Ad.mda-7-induced apoptosis. Oncogene 24: 585-596, 2005.

14. Su ZZ, Lebedeva IV, Sarkar D, et al: Melanoma differentiation associated gene-7, mda-7/IL-24, selectively induces growth suppression, apoptosis and radiosensitization in malignant gliomas in a p53-independent manner. Oncogene 22: 1164-1180, 2003.
15. Yacoub A, Mitchell C, Hong Y, et al: MDA-7 regulates cell growth and radiosensitivity in vitro of primary (non-established) human glioma cells. Cancer Biol Ther 3: 739-751, 2004.

16. Lebedeva IV, Su ZZ, Sarkar D, et al: Melanoma differentiation associated gene-7, mda-7/interleukin-24, induces apoptosis in prostate cancer cells by promoting mitochondrial dysfunction and inducing reactive oxygen species. Cancer Res 63: 8138-8144, 2003.

17. Su Z, Lebedeva IV, Gopalkrishnan RV, et al: A combinatorial approach for selectively inducing programmed cell death in human pancreatic cancer cells. Proc Natl Acad Sci USA 98: 10332-10337, 2001.

18. Lebedeva IV, Su ZZ, Chang Y, et al: The cancer growth suppressing gene mda-7 induces apoptosis selectively in human melanoma cells. Oncogene 21: 708-718, 2002.

19. Mhashilkar AM, Schrock RD, Hindi M, et al: Melanoma differentiation associated gene-7 (mda-7): a novel anti-tumor gene for cancer gene therapy. Mol Med 7: 271-282, 2001.

20. Tian H, Wang J, Zhang B, et al: MDA-7/IL-24 induces Bcl-2 denitrosylation and ubiquitin-degradation involved in cancer cell apoptosis. PLoS One 7: e37200, 2012.

21. Kawabe S, Nishikawa T, Munshi A, et al: Adenovirus-mediated mda-7 gene expression radiosensitizes non-small cell lung cancer cells via TP53-independent mechanisms. Mol Ther 6: 637-644, 2002.

22. Fan H, Suzuki T, Ogata M, et al: Expression of PCNA, ICAM-1, and vimentin in lens epithelial cells of cataract patients with and without type 2 diabetes. Tokai J Exp Clin Med 37: 51-56, 2012.

23. Matrisian LM: The matrix-degrading metalloproteinases. Bioessays 14: 455-463, 1992.

24. Shapiro SD: Matrix metalloproteinase degradation of extracellular matrix: biological consequences. Curr Opin Cell Biol 10: 602-608, 1998

25. Nakopoulou L, Tsirmpa I, Alexandrou P, et al: MMP-2 protein in invasive breast cancer and the impact of MMP-2/TIMP-2 phenotype on overall survival. Breast Cancer Res Treat 77: 145-155, 2003.

26. Zhao XL, Sun T, Che N, et al: Promotion of hepatocellular carcinoma metastasis through matrix metalloproteinase activation by epithelial-mesenchymal transition regulator Twist1. J Cell Mol Med 15: 691-700, 2011.

27. Sun T, Zhao N, Zhao XL, et al: Expression and functional significance of Twistl in hepatocellular carcinoma: its role in vasculogenic mimicry. Hepatology 51: 545-556, 2010.

28. Kang MH, Oh SC, Lee HJ, et al: Metastatic function of BMP-2 in gastric cancer cells: The role of PI3K/AKT, MAPK, the NF- $\mathrm{BB}$ pathway, and MMP-9 expression. Exp Cell Res 317: 1746-1762, 2011.

29. Lu JT, Zhao WD, He W and Wei W: Hedgehog signaling pathway mediates invasion and metastasis of hepatocellular carcinoma via ERK pathway. Acta Pharmacol Sin 33: 691-700, 2012. 